\title{
Synchronization Control of Large Crawler Crane Driven by Double Winches Using Hook Angle Feedback Information
}

\author{
Jun Yao*, Yu Tang and Zhencai Zhu \\ School of Mechatronic Engineering, China University of Mining and Technology, Xuzhou 221116, People's Republic of \\ China
}

\begin{abstract}
During the operation of a crawler crane driven by double winches, it is important to make the two winches actuate synchronously so that the hook is in a horizontal state to prevent accidents. In this paper, a novel synchronization control strategy for crawler crane driven by double winches using hook angle feedback information is proposed. The hook angle proportional to the length error of ropes is measured by a wireless angle sensor firstly and is then employed as a feedback control signal. To further improve the synchronization performance, cross-coupled control scheme together with the variable speed PID control is utilized on the basis of the collected hook angle signal. Simulations and experiments are then conducted and the results demonstrate that the proposed control scheme can obtain a better synchronization performance than the conventional control strategy using encoders and the inclination of the hook is greatly reduced to a limited small range.
\end{abstract}

Keywords: Crawler crane, cross-coupled control, synchronization control, variable speed PID control.

\section{INTRODUCTION}

Crawler cranes are essential facilities for lifting heavy equipment and are extensively applied in the construction of industry electric power, bridges, petrochemical engineering, water conservancy and hydropower, etc. $[1,2]$. With the increasing lifting tonnage of crawler cranes, the lifting system driven by one winch cannot provide sufficient driving torque, and therefore multi winches are employed to overcome this shortcoming. Currently, the most widely used multi winches system in crawler cranes is the double winches system, which takes advantage of two wench mechanisms to lift or descend the hook so as to finish the hoisting operation, and the key issue that must be considered during the whole process is to keep the two winches actuating synchronously [1-3].

However, due to the reasons of pressure surge, leakage, disturbance of payload, structural difference of motors, nonlinear friction, etc., the synchronization performance of the two winches is greatly affected, and results in the inclination of the hook, which further accelerates the abrasion of steel ropes. Besides, when a crawler crane operates with an inclined hook, the load applied to the two winches is not coincident, and may cause the overloading of winches and may give rise to accidents. So, the synchronization of winches to level the hook during the hoisting process is closely related with the safety of crawler cranes.

The synchronization control of crawler cranes belongs to the multi-axis control field [4], and many multi-axis control strategies, such as master-slave control [5], cross-coupled

*Address correspondence to this author at the School of Mechatronic Engineering, China University of Mining and Technology, Xuzhou 221116, People's Republic of China; Tel: 13805216686; E-mail: cumtid@163.com control [6], extended cross-coupled control [7], adaptive control [8], etc., which can be utilized to evaluate the synchronization of winches, and all these control strategies are aimed at the synchronization of revolving speed with obtaining feasible results. However, as for crawler cranes, the synchronization of revolving speed cannot guarantee the synchronization of rope lengths due to the fact that the layers and loops of the ropes, errors of the two roller radius, rope elastic extension with different loads, etc., are not taken into consideration, and therefore even a high accuracy synchronization of revolving speed of winches is obtained. The hook lifting payload still cannot retain the horizontal position during the operation process.

To solve these problems, this paper proposes a novel synchronization control strategy for crawler cranes using the hook angle feedback information. The contributions are organized as follows. The conventional and proposed control strategy for crawler cranes is discussed in detail in section 2. Experiments are conducted in section 3 to demonstrate the effectiveness of the proposed strategy. Section 4 concludes the main points and contributions.

\section{CONTROLLER DESIGN}

\subsection{Conventional Synchronization Control Strategy Using Encoders}

The conventional synchronization control strategy is presented in Fig. (1). As shown in the figure, the same command signal is firstly sent to the variable pumps and motors to control their displacements so as to ensure the rough synchronization of the two motors, and then two rotary encoders are utilized to measure the rotational angles of the hoist rollers. The lengths of the hoist ropes are obtained by multiplying the measured angles with the 
average radius of the rollers, and therefore the length error between the two hoist ropes is calculated. With the estimated length error, a PID controller is exploited to further improve the synchronization accuracy through compensating the velocity of the second variable pump. The control input to the second pump can be written as:

$u=u_{1}+K_{p} \Delta L+K_{i} \int \Delta L d t+K_{d} \frac{d \Delta L}{d t}$

where $u_{1}$ is the command signal to the variable pump, $\Delta L$ is the length error when the rollers are twining or releasing the hoist ropes, $K_{p}$ is the proportional coefficient, $K_{i}$ is the integral coefficient, and $K_{d}$ is the differential coefficient.

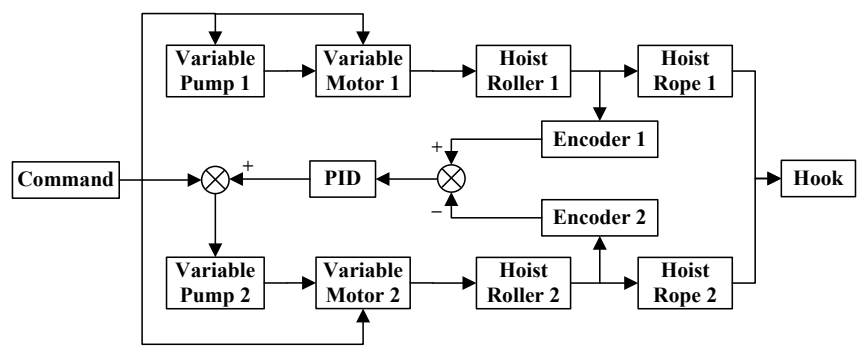

Fig. (1). Conventional synchronization control strategy for crawler crane.

Due to the fact that the command signal to the pumps and motors is open loop control strategy, the synchronization accuracy is firstly affected by the control scheme. Besides, the adopted velocity compensation PID control policy is a master-slave method, and the tracking error always occurs between the master and the slave actuator. Another important issue needs to be accounted is that the calculated length error between the two hoist ropes using encoders is approximate, which neglects the layers and loops of the ropes, errors of the two roller radius, rope elastic extension with different loads, etc. Therefore, the conventional synchronization with encoders is not a feasible control method, and the control accuracy is poor.

\subsection{Proposed Synchronization Control Strategy Using Hook Angle Feedback}

\subsubsection{Relation Between Hook Angle and Length Error}

Fig. (2) depicts the scheme for analyzing the relation between hook angle and length error of crawler crane driven by double winches. As shown in the figure, $A_{0}$ is the upper suspension point of the left hoist rope, $B_{0}$ is the upper suspension point of the right hoist rope, $A_{1}$ is the connecting point between the left rope and hook, $B_{1}$ is the connecting rope between the right rope and the hook, $O$ is the centre of rotation when the hook inclines, $A_{2}$ and $B_{2}$ are the connecting points of the inclined hook, $\theta$ is the inclined angle of the hook.

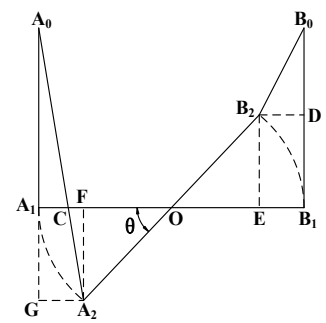

Fig. (2). Scheme of hook angle and rope length error.

Let $\overline{A_{0} A_{1}}=\overline{B_{0} B_{1}}=l_{0}$ and $\overline{O A_{1}}=\overline{O B_{1}}=\overline{O A_{2}}=\overline{O B_{2}}=l_{1}$, the following equations can be concluded

$\left\{\begin{array}{l}\overline{F A_{2}}=\overline{E B_{2}}=l_{1} \sin \theta \\ \overline{F A_{1}}=\overline{E B_{1}}=l_{1}-l_{1} \cos \theta\end{array}\right.$

Employing the Pythagorean theorem, we get

$$
\begin{aligned}
& \overline{A_{0} A_{2}}=\sqrt{\left(\overline{A_{0} A_{1}}+\overline{A_{1} G}\right)^{2}+\left(\overline{A_{2} G}\right)^{2}}, \\
& =\sqrt{\left(l_{0}+l_{1} \sin \theta\right)^{2}+\left(l_{1}-l_{1} \cos \theta\right)^{2}} \\
& \overline{B_{0} B_{2}}=\sqrt{\left(\overline{B_{0} B_{1}}-\overline{B_{1} D}\right)^{2}+\left(\overline{B_{2} D}\right)^{2}} \\
& =\sqrt{\left(l_{0}-l_{1} \sin \theta\right)^{2}+\left(l_{1}-l_{1} \cos \theta\right)^{2}} .
\end{aligned}
$$

Combining Equation (3) and (4), the length error between the ropes of inclined hook can be deduced as

$$
\begin{aligned}
\Delta l & =\overline{A_{0} A_{2}}-\overline{B_{0} B_{2}} \\
& =\sqrt{\left(l_{0}+l_{1} \sin \theta\right)^{2}+\left(l_{1}-l_{1} \cos \theta\right)^{2}} \\
& -\sqrt{\left(l_{0}-l_{1} \sin \theta\right)^{2}+\left(l_{1}-l_{1} \cos \theta\right)^{2}}
\end{aligned}
$$

During the actual operating process, to ensure the safety of the crawler crane, the inclination of the hook must be retained in a limited range, and when the inclination angle is small, the following equations hold

$\left\{\begin{array}{l}\sin \theta \approx \theta \\ \cos \theta \approx 1\end{array}\right.$.

Substituting Equation (6) into Equation (5) and simplifying, we get

$$
\begin{aligned}
\Delta l & \approx \sqrt{\left(l_{0}+l_{1} \theta\right)^{2}+\left(l_{1}-l_{1}\right)^{2}}-\sqrt{\left(l_{0}-l_{1} \theta\right)^{2}+\left(l_{1}-l_{1}\right)^{2}} \\
& =\left(l_{0}+l_{1} \theta\right)-\left(l_{0}-l_{1} \theta\right)=2 l_{1} \theta
\end{aligned}
$$

As can be seen from Equation (7), the length error between the two ropes appears to be a linear relationship with $l_{1}$ and $\theta$, and for a specified crawler crane whose structural dimensions are fixed, $l_{1}$ is a constant value, and therefore the length error $\Delta l$ is proportional to the inclination angle $\theta$. So, compared with the method by using encoders, if the inclination angle of the hook can be measured in practice, the length error is more accurately reflected by the collected angle. 


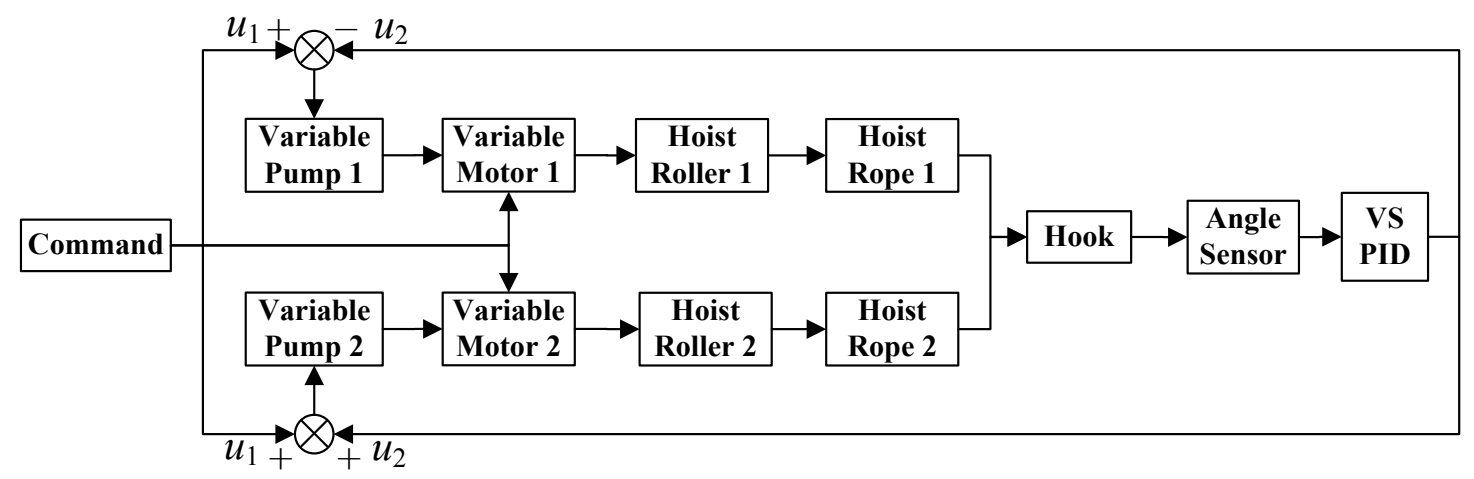

Fig. (3). Proposed synchronization strategy using hook angle feedback.

\subsubsection{Synchronization Strategy Using Hook Angle Feedback}

The proposed synchronization control strategy using hook angle feedback information is demonstrated in Fig. (3). The hook angle is collected by a wireless angle sensor installed on the hook, and to further improve the synchronization accuracy, a cross-coupled control scheme is adopted here to overcome the shortcoming of master-slave method.

As shown in Fig. (3), the same command is firstly employed to the variable pumps and motors to evaluate the synchronization roughly. Then, the hook angle information which directly reflects the length error between the two ropes is collected by the angle sensor as a feedback signal. The feedback angle signal is injected to a VS-PID controller and generates an extra adjusting control signal, which is negative to variable pump 1 , while positive to pump 2 .

From Fig. (3), the control input to variable pump 1 can be expressed as

$u=u_{1}-\left(K_{p} \theta+K_{i} \int f(\theta) \theta d t+K_{d} \frac{d \theta}{d t}\right)$,

and input to variable pump 2 can be written as

$u=u_{1}+\left(K_{p} \theta+K_{i} \int f(\theta) \theta d t+K_{d} \frac{d \theta}{d t}\right)$,

where $u_{1}$ is the command signal to variable pump, $\theta$ is the actual hook angle measured by angle sensor, $K_{p}$ is the proportional coefficient, $K_{i}$ is the integral coefficient, $K_{d}$ is the differential coefficient, $f(\theta)$ is the variable speed integral coefficient and can be given as

$f(\theta)= \begin{cases}1 & \text { if } \theta \leq b_{L} \\ \frac{b_{U}-|\theta|}{b_{U}-b_{L}} & \text { if } b_{L}<\theta \leq b_{U}, \\ 0 & \text { if } \theta>b_{U}\end{cases}$

where $b_{U}$ and $b_{L}$ are the upper and lower bounds of absolute hook angle. The benefit of utilizing variable speed integral lies in the fact that the integral coefficient reduces to zero gradually when the hook angle is large and the coefficient increases to one when the angle is small, which ensures a short adjusting time and a good stability of the system.

\section{EXPERIMENTAL RESULTS}

To further demonstrate the effectiveness of the proposed synchronization control strategy for large crawler cranes, experiments are conducted on a specified crawler crane and the experimental setup is given in Fig. (4). As can be seen from the figure, a wireless angle sensor is attached on the hook to measure the inclined angle of the hook so as to implement the proposed angle feedback control policy.

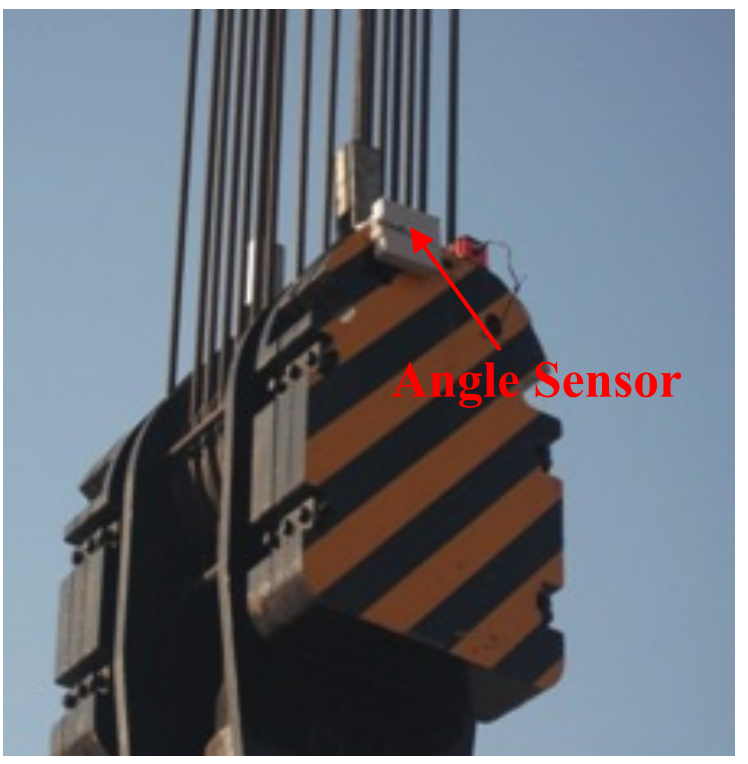

Fig. (4). Experimental setup for the synchronization control of crawler crane.

Firstly, experiments using conventional synchronization control strategy with encoders are conducted and the experimental results are presented in Fig. (5). From the figure, it can be easily found that during the lifting and descending process of the crawler crane, when using the conventional synchronization strategy, although the initial state of the hook is horizontal, the hook inclination angle increases over time and the final results are not acceptable. Therefore, the conventional control strategy using encoders 


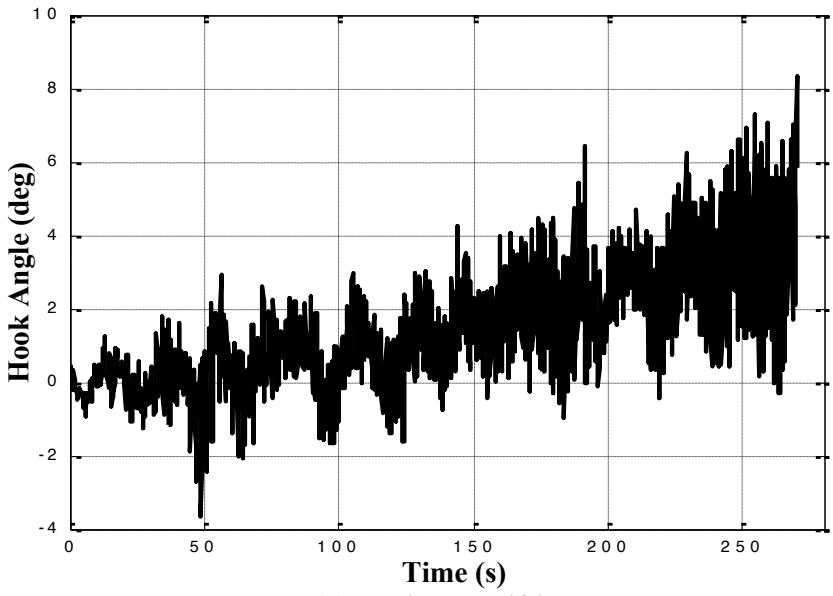

(a) Horizontal lifting

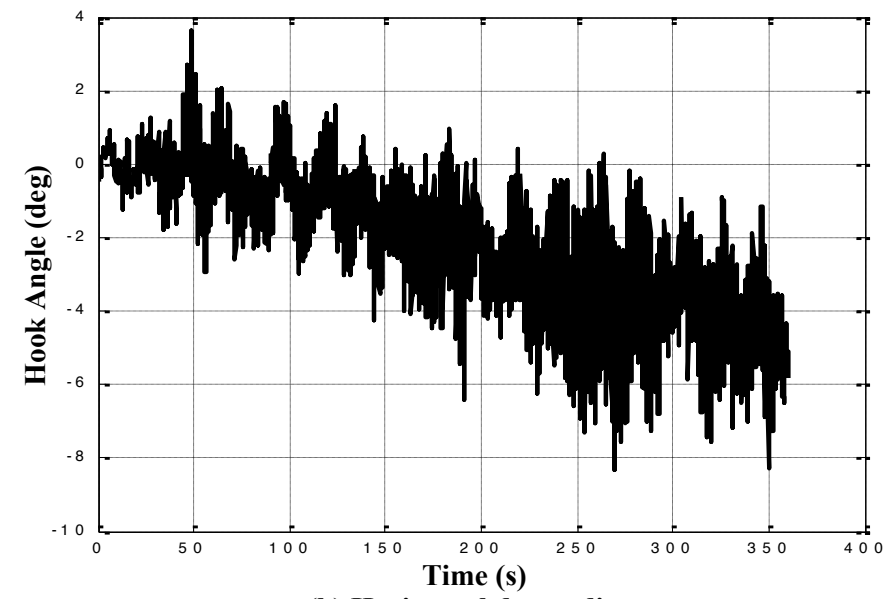

(b) Horizontal descending

Fig. (5). Conventional synchronization strategy using encoders.

cannot evaluate the synchronization of crawler cranes with high accuracy.

To verify the correctness of the proposed strategy using hook angle information, the working conditions without payload are tested, and hook angle changes during the lifting and descending process of the crawler crane as depicted in Fig. (6). It can be easily concluded from the figure, when using the proposed strategy, for the case of initially horizontal lifting, the hook angle during the whole process is retained in $-3.6^{\circ} \sim 0.6^{\circ}$, and for the horizontal descending

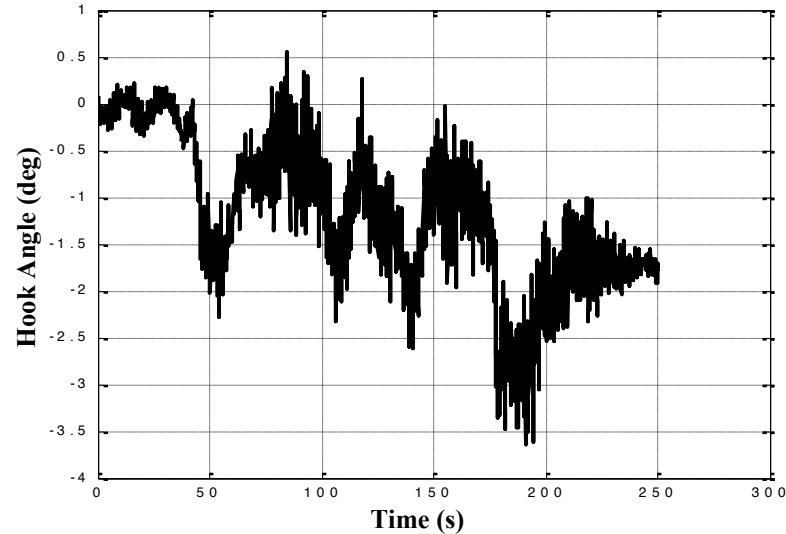

(a) Horizontal lifting

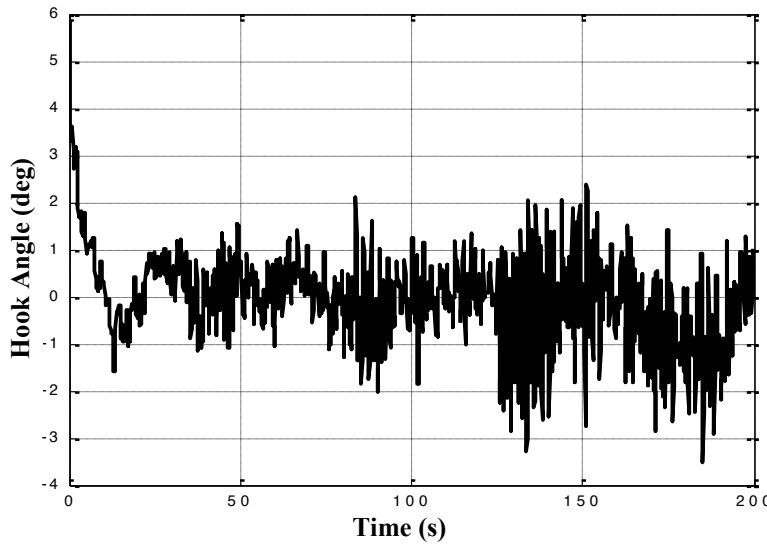

(c) Inclined lifting case, it is $-2.4^{\circ} \sim 4.0^{\circ}$. As for the initially inclined cases, the hook angle is kept within $-3.5^{\circ} \sim+2.4^{\circ}$ after $5 \mathrm{~s}$ adjustment during the lifting process and $-3.6^{\circ} \sim+3.4^{\circ}$ after $18 \mathrm{~s}$ adjustment for the descending condition. All these results demonstrate that the proposed scheme is feasible for the synchronization control of crawler cranes without payload.

In addition, the working condition with payload is also taken into consideration and the experimental results are presented in Fig. (7). The first picture in Fig. (7) corresponds to the initially horizontal lifting process with payload and the

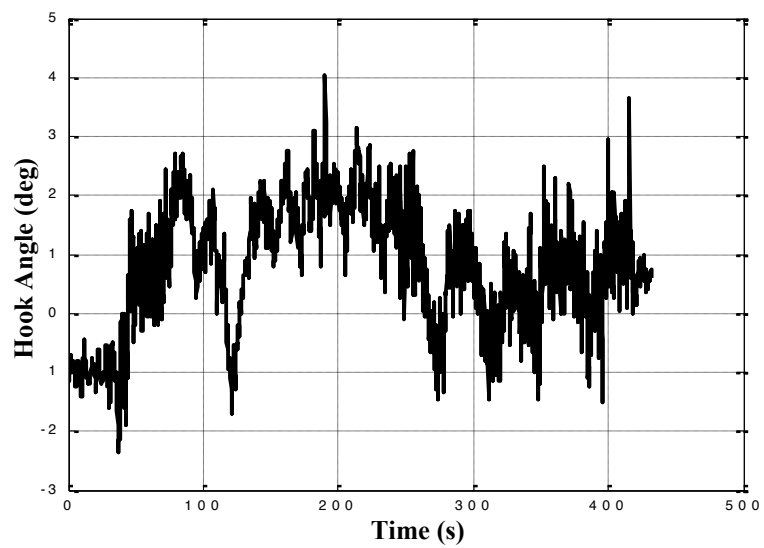

(b) Horizontal descending

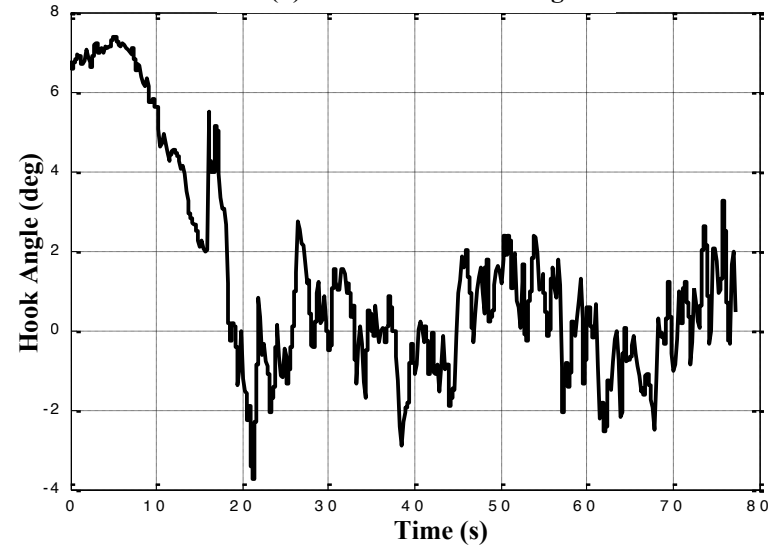

(b) Inclined descending

Fig. (6). Proposed synchronization strategy using hook angles without payload. 


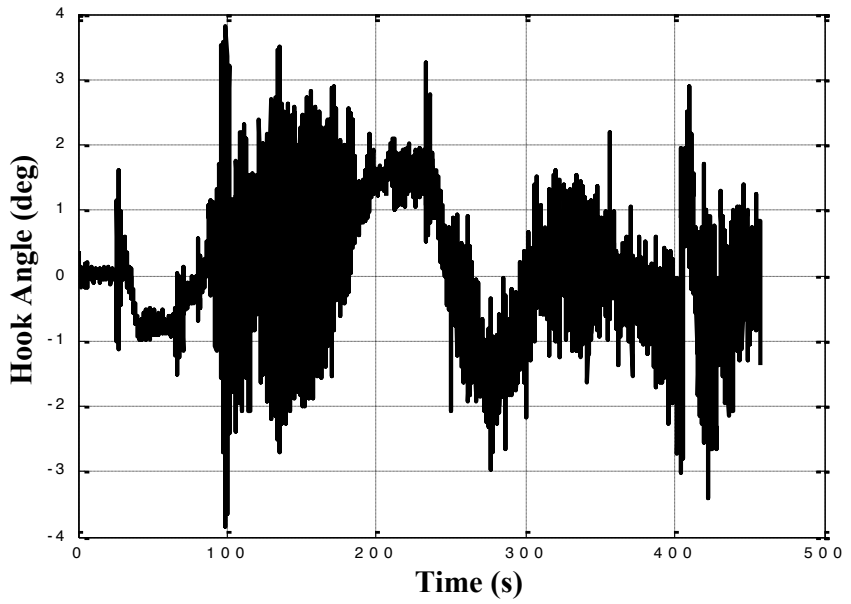

(a) Horizontal lifting

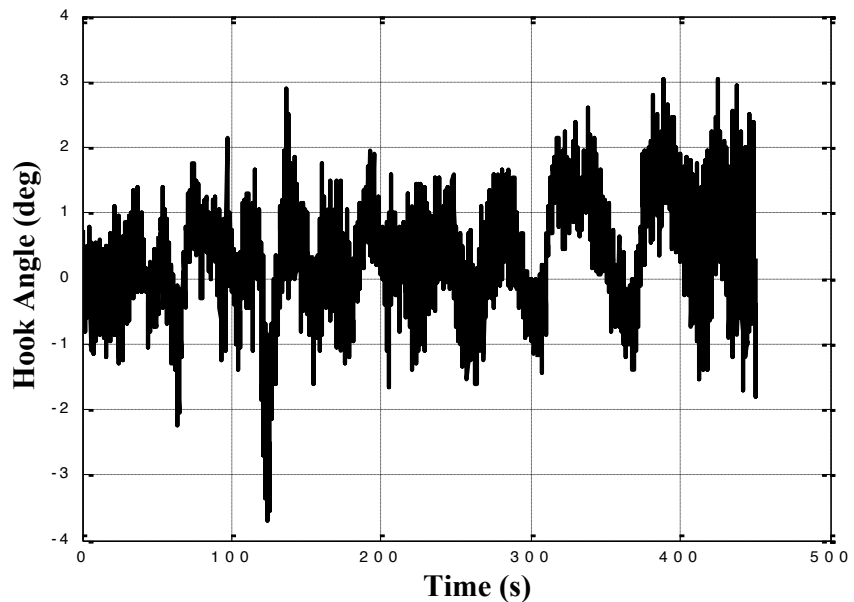

(b) Horizontal descending

Fig. (7). Proposed synchronization strategy using hook angles with payload.

second one is the initially horizontal descending case. From the two figures, it can easily be found that when lifting or descending a payload, compared with the condition without payload, the change for the hook angle during the whole operation process is larger, but with the proposed strategy, the hook angle can still be retained within the range of $\pm 4^{\circ}$, which also shows a satisfactory synchronization performance of the crawler crane.

\section{CONCLUSION}

In this paper, to improve the synchronization performance of crawler cranes driven by double winches, a control strategy using the hook angle feedback information is adopted, and theoretical and experimental analyses are conducted to verify the effectiveness of the proposed scheme. From the detailed analysis, the following conclusions are drawn:

(1) The hook angle measured by the wireless sensor is proportional to the length error of the ropes for a specified crawler crane, and thus can be utilized as a feedback signal for the synchronization control of crawler cranes;

(2) Compared with the conventional synchronization strategy using encoders to measure the length error, the proposed cross-coupled variable speed PID controller using hook angle feedback information exhibits a better control performance and the inclination of the hook is greatly reduced to a limited small range.

\section{CONFLICT OF INTEREST}

The authors confirm that this article content has no conflict of interest.

\section{ACKNOWLEDGEMENTS}

This research was supported by the National High Technology Research and Development (863) Program of China "Research and application of key technologies of thousand tonnage large crawler cranes" (grant number 2012AA041804).

\section{REFERENCES}

[1] L. Z. Ma, J. Liu, X. L. Diao, M. L. Dong, "The elastic dynamic analysis of crawler crane boom system," Advanced Materials Research, vol. 482, pp. 1138-1141, 2012.

[2] T. Jiang, G. Y. Xue, and Y. P. You, "Study on the impact of wind load on large crawler crane typical truss structure," Advanced Materials Research, vol. 295, pp. 2133-2136, 2011.

[3] H. Araya, M. Kakuzen, H. Kinugawa, and T. Arai, "Level luffing control system for crawler cranes," Automation in Construction, vol. 13, no. 5, pp. 689-697, 2004.

[4] S. S. Yeh, and P. L. Hsu, "Analysis and design of integrated control for multi-axis motion systems," IEEE Transactions on Control Systems Technology, vol. 11, no. 3, pp. 375-382, 2003.

[5] Y. Yokokohji, and T. Yoshikawa, "Bilateral control of master-slave manipulators for ideal kinesthetic coupling-formulation and experiment," IEEE Transactions on Robotics and Automation, vol. 10, no. 5, pp. 605-620, 1994.

[6] Y. Koren, "Cross-coupled biaxial computer control for manufacturing systems," Journal of Dynamic Systems, Measurement, and Control, vol. 102, no. 4, pp. 265-272, 1980.

[7] G. Turl, M. Summer, and G. A. Asher, "Synchronized multi-motor control system using sensorless induction motor drives," In: Proceedings of International Conference on Power Electronics, Machines and Drives," Institution of Electrical Engineers, London, pp. 38-43, 2002.

[8] M. Tomizuka, J.S. Hu, T.C. Chiu, and T. Kamano, "Synchronization of two motion control axes under adaptive feedforward control," Journal of Dynamic Systems, Measurement, and Control, vol. 114, no. 2, pp. 196-203, 1992. 